Proceedings of the 48th International School and Conference on the Physics of Semiconductors "Jaszowiec 2019"

\title{
Influence of Electron Blocking Layer on Properties of InGaN-Based Laser Diodes Grown by Plasma-Assisted Molecular Beam Epitaxy
}

\author{
M. Hajdel ${ }^{a} *$, G. Muziol ${ }^{a}$, K. Nowakowski-SzkudlareK ${ }^{a}$, M. SieKACZ $^{a}$, \\ A. Feduniewicz-Żmuda ${ }^{a}$, P. WOLnY ${ }^{a}$ And C. Skierbiszewski ${ }^{a, b}$ \\ ${ }^{a}$ Institute of High Pressure Physics, Polish Academy of Sciences, \\ Sokołowska 29/37, PL-01142 Warsaw, Poland \\ ${ }^{b}$ TopGaN Ltd, Sokołowska 29/37, PL-01142 Warsaw, Poland
}

\begin{abstract}
We investigate influence of $\mathrm{Mg}$ doping concentration and the thickness of electron blocking layer on properties of InGaN-based laser diodes grown by plasma-assisted molecular beam epitaxy. Using simple measurements of light-current characteristics and simulations, two main conclusions are drawn. First one - the $\mathrm{Mg}$ dopant is responsible for optical losses, as in the case of the laser diodes grown by metalorganic vapor phase epitaxy. Second one - the low $\mathrm{Mg}$ doping causes electrons to overflow through electron blocking layer. Additionally, tunneling is proposed as an escape mechanism of carriers from active region for thin electron blocking layer.
\end{abstract}

DOI: 10.12693/APhysPolA.136.593

PACS/topics: InGaN lasers, optical losses, Mg doping, EBL thickness, PAMBE

\section{Introduction}

InGaN based laser diodes (LDs) are attracting a lot of attention as efficient light emitters for displays, data storage, and general lighting [1]. The crucial parameter that limits the output power of III-nitride LDs are the internal optical losses $\left(\alpha_{i}\right)$. First InGaN LDs suffered from high $\alpha_{i} \approx 40 \mathrm{~cm}^{-1}[2,3]$ and because of that the maximum values of optical power in the order of $100 \mathrm{~mW}$. Nowadays, state of the art LDs have $\alpha_{i}=1 \mathrm{~cm}^{-1}$ and the maximum optical power is $7.2 \mathrm{~W}$ [4]. It was shown that, in the case of InGaN-based LDs, the high $\alpha_{i}$ originates from light absorption in the $\mathrm{Mg}$-doped layers [5-11].

Calculation of Kioupakis et al. have shown that absorption cross-section of $\mathrm{Si}$ and $\mathrm{Mg}$ do not differ much. However, the higher concentrations of $\mathrm{Mg}$ dopant used in III-nitride LDs might have the dominant impact on large $\alpha_{i}$ values $[9,10]$. Due to high activation energy of $\mathrm{Mg}$ atoms, the concentration of $\mathrm{Mg}$ has to be high enough to ensure sufficient electrical conductivity of $p$-type layers. In case of III-nitride LDs the most highly Mg-doped layer is the electron blocking layer (EBL). It is introduced after the active region to block the overflow of electrons that comes from the difference in mobility of electrons and holes in GaN $[12,13]$. Without this Mg-doped layer the injection efficiency $\left(\eta_{i}\right)$ of carriers into the active region would be low, and the overall efficiency of the LD will drop. It was shown that in order to reduce the total $\alpha_{i}$ one can introduce an undoped layer placed between

*corresponding author; e-mail: hajdel@unipress.waw.pl active region and $p$-type doped layers $[8,11]$. It decreases the optical confinement factor of $\mathrm{Mg}$-doped layers $\left(\Gamma_{\mathrm{Mg}}\right)$ and thus decreases the $\alpha_{i}$ leading to an improvement in threshold current density $\left(j_{t h}\right)$ and slope efficiency (SE). However, after a certain thickness of the undoped layer is reached the $\eta_{i}$ starts to drop leading to an interplay between $\alpha_{i}$ and $\eta_{i}$. This makes the designing of $p$-type layers a crucial issue for developing high performance LD.

Majority of the papers have reported the LDs grown by the metalorganic vapor phase epitaxy (MOVPE) technique. Recent advancement in the plasma assisted molecular beam epitaxy (PAMBE) technology in the growth of III-nitride LDs, especially the demonstration of LDs with the lifetime of 100000 hours [14], allows us to answer the question whether the $\mathrm{Mg}$ dopant also causes optical losses in PAMBE-grown LDs. Maybe there are additional sources of absorption present only in MOVPE-grown or in PAMBE-grown LDs. Answer to these questions should verify whatever the current understanding of sources of absorption in high power LDs is correct.

In this paper we experimentally investigate the role of Mg-doping level and thickness of EBL on InGaN-based LD parameters such as $j_{t h}$ and SE. Based on experimental data and simulations, a model describing the impact of $\mathrm{Mg}$ on $\alpha_{i}$ and $\eta_{i}$ is also proposed.

\section{Experimental}

The LDs were grown in metal-rich conditions by PAMBE, using a VG V90 reactor, on commercially available LUMILOG GaN substrates with threading dislocation densities of the order of $(1-5) \times 10^{7} \mathrm{~cm}^{-2}$. $\mathrm{LD}$ structure is presented in Fig. 1a. It consists of $700 \mathrm{~nm}$ $\mathrm{Al}_{0.07} \mathrm{Ga}_{0.93} \mathrm{~N}$ :Si bottom cladding and $100 \mathrm{~nm}$ GaN:Si layer with Si concentration of $2 \times 10^{18} \mathrm{~cm}^{-3}$. Bottom and 

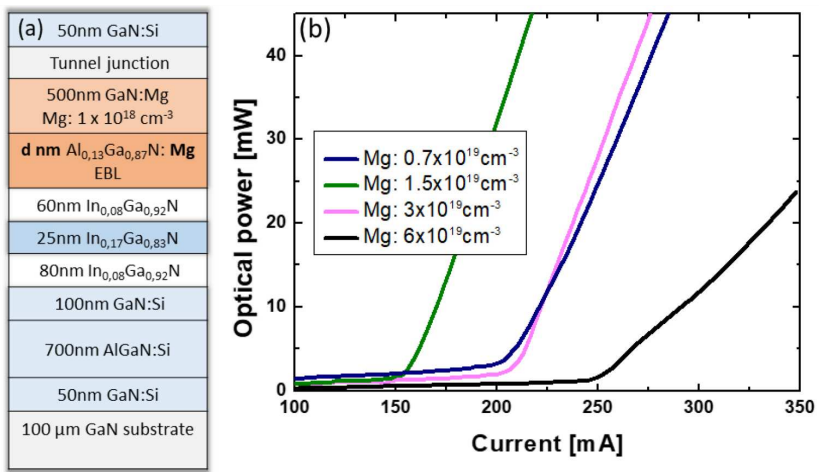

Fig. 1. (a) Structure of studied LDs. The doping and thickness of EBL are varied. (b) Exemplary L-I characteristics of measured LDs.

upper waveguides are undoped $\operatorname{In}_{0.08} \mathrm{Ga}_{0.92} \mathrm{~N}$ layers with thickness of $80 \mathrm{~nm}$ and $60 \mathrm{~nm}$, respectively. The active region is a $25 \mathrm{~nm}$ thick $\operatorname{In}_{0.17} \mathrm{Ga}_{0.83} \mathrm{~N}$ quantum well [15]. At the end of the InGaN waveguide the EBL consisting of $\mathrm{Al}_{0.13} \mathrm{Ga}_{0.85} \mathrm{~N}: \mathrm{Mg}$ is placed. Upper cladding is a $500 \mathrm{~nm} p$-type $\mathrm{GaN}$ with $\mathrm{Mg}$ doping concentation of $1 \times 10^{18} \mathrm{~cm}^{-3}$. This is followed by a TJ [16] capped with GaN:Si contact layer. Two sets of samples were realized with different designs of EBL. In the first set, the $\mathrm{Mg}$ doping levels were changed $(0.7,1.5,3,6) \times 10^{19} \mathrm{~cm}^{-3}$ for a $20 \mathrm{~nm}$ thick EBL. In the second set, the doping level was constant at $1.5 \times 10^{19} \mathrm{~cm}^{-3}$ and the thickness was changed, i.e., $(5,10,20) \mathrm{nm}$. More details on growth and processing of the devices can be found in [17].

To study the influence of EBL design on properties of LDs the light-current $(L-I)$ characteristics of grown samples were measured using an integrating sphere. To avoid the heating effects measurements were done under pulse operation with pulse length of $200 \mathrm{~ns}$. Exemplary $L-I$ curves are shown in Fig. 1b. To accurately determine the influence of EBL design in EBL tens of LDs for each sample were measured and $j_{t h}$ and SE values were estimated and then averaged. To obtain insight into the physical processes taking place in the LD the band diagrams and $\eta_{i}$ of grown devices were simulated using SiLENSe software [18]. Optical mode distribution and confinement factors were calculated using a 1D model [19].

\section{Results and discussion}

\section{1. $M g$ doping concentration in $E B L$}

Averaged values of $j_{t h}$ and SE of LDs with different $\mathrm{Mg}$ doping of EBL are presented in Fig. 2a and b, respectively. The error bars depict the standard deviation among the measured devices. For the highest $\mathrm{Mg}$ doping the $j_{t h}$ and SE are equal to $4.62 \mathrm{kA} \mathrm{cm}^{-2}$ and $0.359 \mathrm{~W} / \mathrm{A}$, respectively. When the $\mathrm{Mg}$ doping is lowered, both $j_{t h}$ and SE show an improvement. To understand how $\mathrm{Mg}$ influences the internal properties of LDs the following model has been created. The $j_{t h}$ and SE are related to the internal properties by

$$
\begin{aligned}
& j_{t h}=\frac{\alpha_{i}+\alpha_{m}}{\Gamma \eta_{i}\left(\frac{\mathrm{d} g}{\mathrm{~d} j}\right)}+j_{\mathrm{trans}}, \\
& S E=\eta_{i}\left(\frac{\alpha_{m}}{\alpha_{i}+\alpha_{m}}\right) \frac{h v}{q},
\end{aligned}
$$

where $\alpha_{m}$ are mirror losses, $\Gamma$ is the confinement factor of active region, $g$ is the material gain, $j_{\text {trans }}$ is the transparency current, $v$ is the frequency of emitted light from device, $q$ is the elementary charge, and $h$ is the Planck constant.

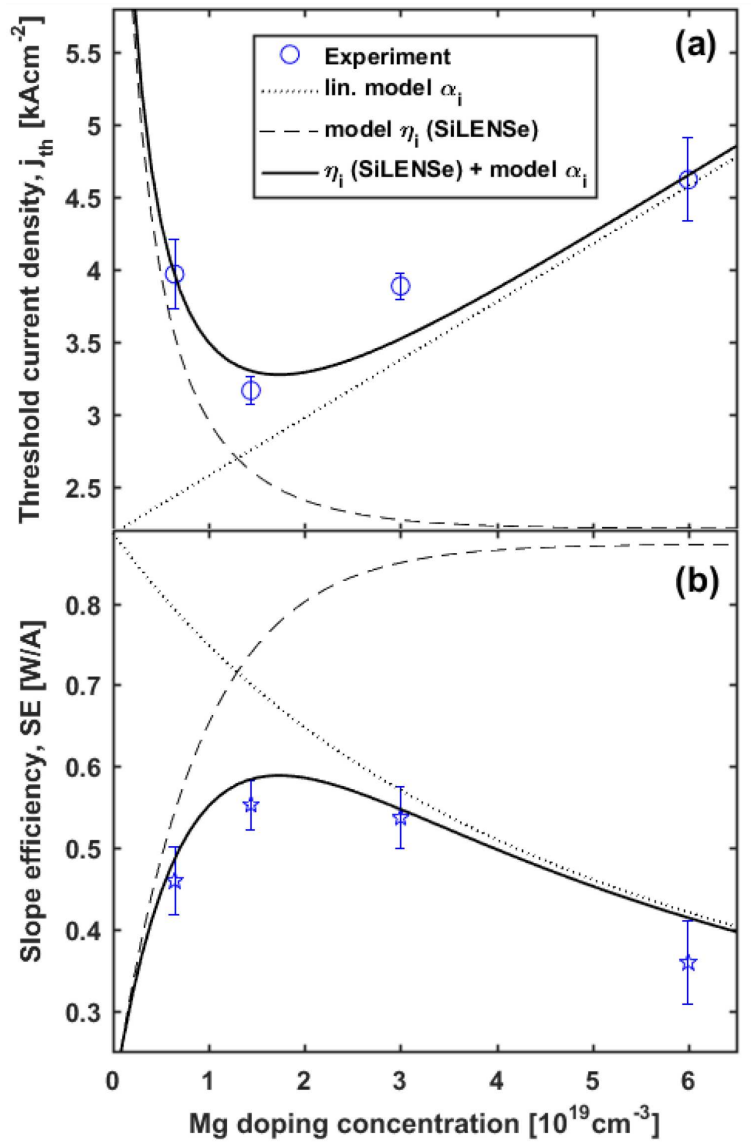

Fig. 2. Dependence of: (a) threshold current density and (b) slope efficiency on $\mathrm{Mg}$ doping level in EBL.

To model the impact of $\mathrm{Mg}$ doping on optical losses the optical confinement with EBL $\left(\Gamma_{\mathrm{EBL}}\right)$ has been calculated. Figure 3 presents the optical mode distribution with the shaded area representing the part of the optical mode involved in light absorption in EBL. The calculated value of $\Gamma_{\mathrm{EBL}}$ is $4.37 \%$. A linear dependence between the absorption and $\mathrm{Mg}$ concentration has been assumed by a relation $\alpha_{\mathrm{Mg}}=112 \mathrm{~cm}^{-2} \times \mathrm{Mg}$ concentration (in units of $10^{19} \mathrm{~cm}^{-3}$ ). Additionally, residual optical losses of $\alpha_{0}=9.5 \mathrm{~cm}^{-1}$ are fitted. The calculated dependence is presented in Fig. 2 as a dotted line. As can be seen, the change of $j_{t h}$ and SE in the regime of high Mg doping $\left(\mathrm{Mg}>2 \times 10^{19} \mathrm{~cm}^{-3}\right)$ can be explained solely by 


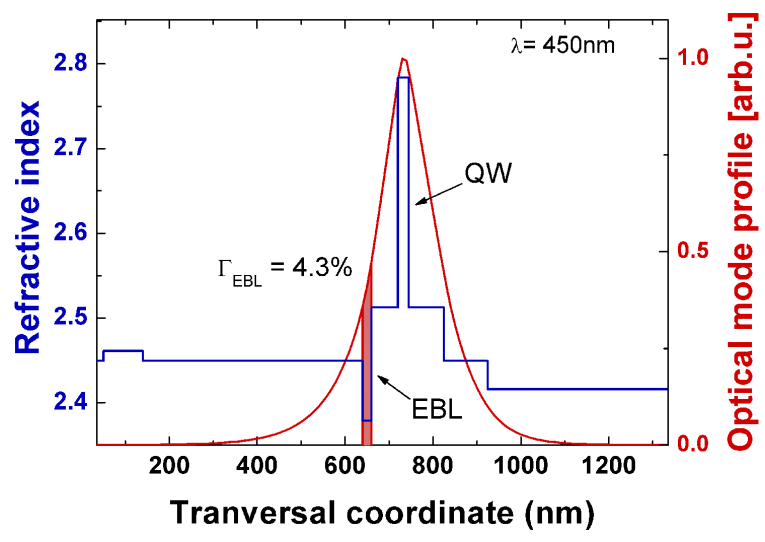

Fig. 3. Refractive index profile and optical mode distribution of studied LDs. Shaded section correspond to the $20 \mathrm{~nm}$ EBL.

the change in the optical losses due to absorption on $\mathrm{Mg}$. However, for lower Mg concentrations a growing discrepancy between this model and the experimental data is observed.

To understand this behavior we have used the SiLENSe 5.4 package [18] equipped with a one dimensional drift diffusion model. Figure 4 presents the calculated conduction band profile of the LDs with $\mathrm{Mg}$ doping of $0.7 \times 10^{19}$ and $6.0 \times 10^{19} \mathrm{~cm}^{-3}$. Only the vicinity of the $\mathrm{QW}$ and EBL is shown. As can be seen the change of the doping in EBL strongly changes the energetic barrier $\left(E_{B}\right)$ in the conduction band which is used to prevent the electron overflow to the $p$-type region. The value of $E_{B}$ decreases from 204 to $105 \mathrm{meV}$ when the $\mathrm{Mg}$ doping is changed from $6.0 \times 10^{19}$ to $0.7 \times 10^{19} \mathrm{~cm}^{-3}$. Such a change affects the injection efficiency. Figure 5 presents the calculated dependence of $\eta_{i}$ on current density for several doping levels and thicknesses. For low current density the $\eta_{i}$ is independent of the EBL design and is equal to 1, which means that all electrons recombine in the QW region. As the current density is increased, a drop in $\eta_{i}$ is observed. This results in a decrease of $\eta_{i}$ for low $\mathrm{Mg}$ doping as it is shown in the inset in Fig. 5 for a fixed current density of $3 \mathrm{kAcm}^{-2}$. Taking into account the change of $\eta_{i}$ with $\mathrm{Mg}$ and assuming a constant $\alpha_{i}$ the $j_{t h}$ and SE can be well modeled in the low $\mathrm{Mg}$ doping regime as shown by dashed line in Fig. 2. A model combining the change of both $\eta_{i}$ and $\alpha_{i}$ with $\mathrm{Mg}$ is constructed and plotted as a solid line in Fig. 2. This model is in good agreement with the experimental data indicating that both $\eta_{i}$ and $\alpha_{i}$ are affected when the $\mathrm{Mg}$ doping level in the EBL is changed. It is important to note that in the model the same fitting parameters are used to describe both the effects on $j_{t h}$ and SE.

Based on the proposed model an optimal value of doping in the EBL can be predicted. For a considered structure it is equal to $1.8 \times 10^{19} \mathrm{~cm}^{-3}$. It is worth mentioning that for different structure designs this value can vary, especially if the EBL and/or InGaN waveguide composition are changed, affecting $\eta_{i}[20]$.

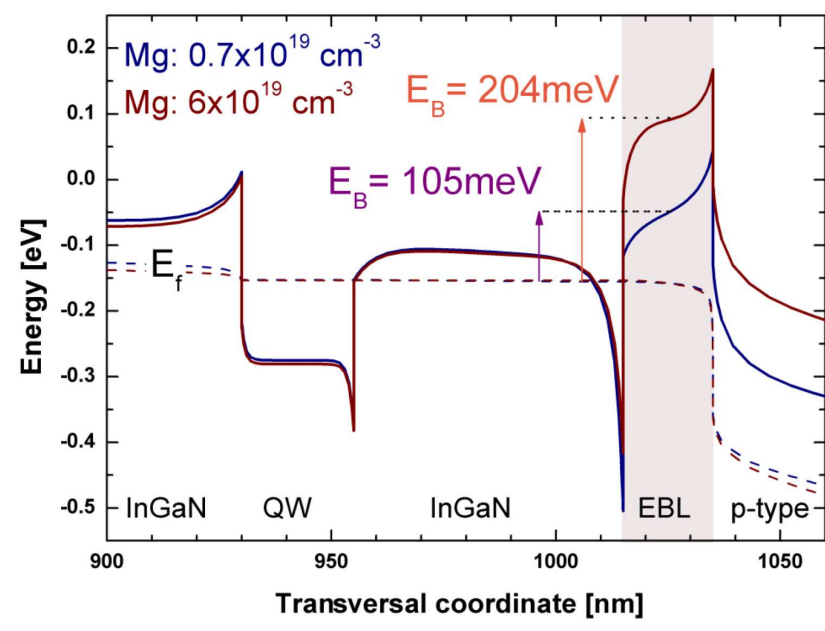

Fig. 4. Calculated conduction band of the LDs with the highest and the lowest $\mathrm{Mg}$ doping concertation.

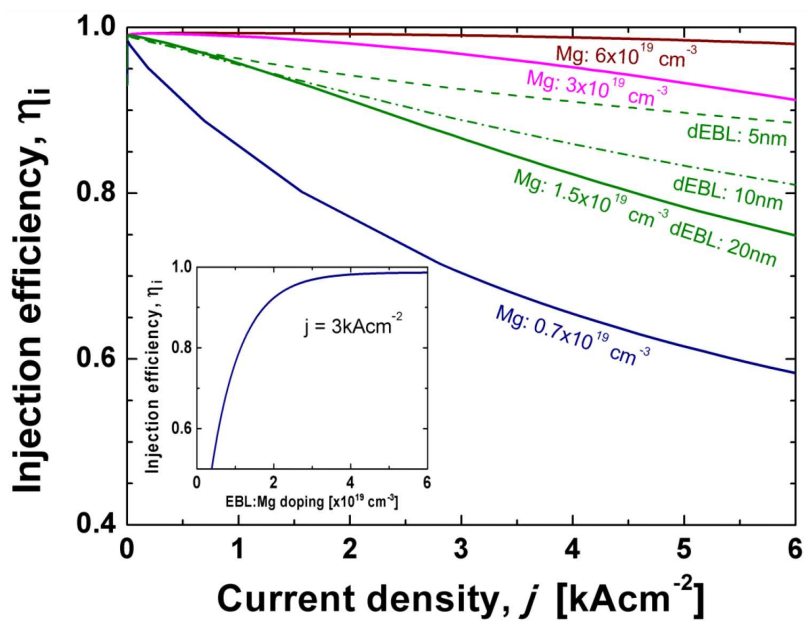

Fig. 5. Simulated dependence of injection efficiency on current density for different $\mathrm{Mg}$ doping level for $20 \mathrm{~nm}$ thick EBL (and for different thicknesses for a constant doping of $1.5 \times 10^{19} \mathrm{~cm}^{-3}$ - dashed and dash-dot lines).

\subsection{EBL thickness}

In the second set of samples the EBL thickness is changed. This should affect the optical losses. The reduction of EBL thickness reduces $\Gamma_{\mathrm{EBL}}$ from $4.37 \%$ to $1.28 \%$ and consequently the $\alpha_{i}$ from $16.8 \mathrm{~cm}^{-1}$ to $11.5 \mathrm{~cm}^{-1}$ for $20 \mathrm{~nm}$ and $5 \mathrm{~nm}$, respectively. The measured $j_{t h}$ and SE are presented in Fig. 6. The decrease of the EBL thickness caused a significant increase of $j_{t h}$ and a decrease of SE. To understand the unexpected change in $j_{t h}$ and SE once again the band profiles and injection efficiency were calculated. In Fig. 7 one can see the conduction band profile. The height of the energetic barrier remains unchanged, however, its width decreases. The calculated current density $\eta_{i}$ is presented in Fig. 5. Its slight increase can be seen when the EBL thickness is reduced. 


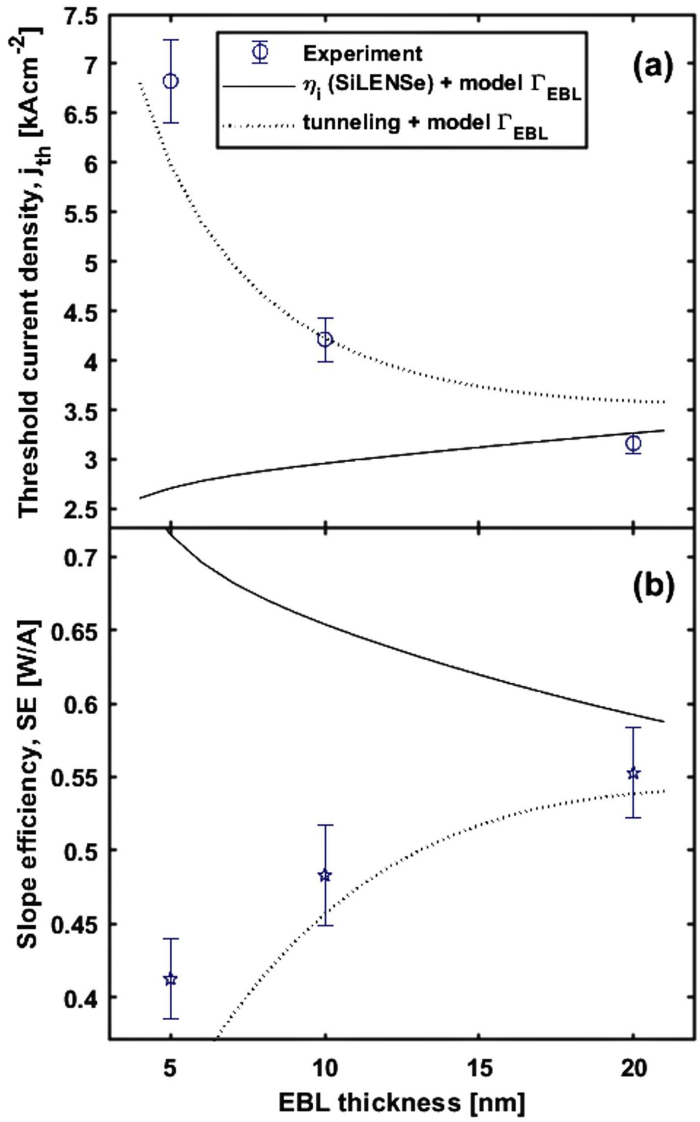

Fig. 6. Dependence of: (a) threshold current density and (b) slope efficiency on EBL thickness.

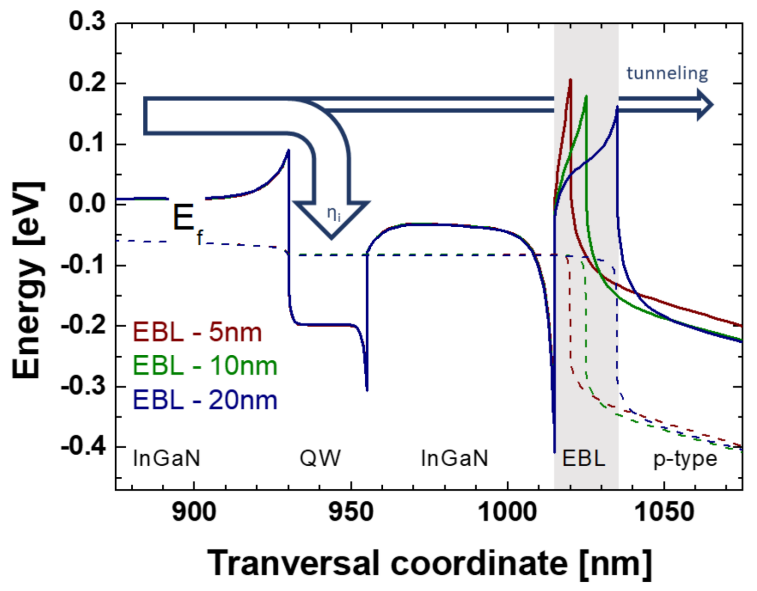

Fig. 7. Calculated conduction band of the LDs with the EBL thickness of 5, 10 and $20 \mathrm{~nm}$. Arrows indicate the electron flow paths.

The model describing the calculated change of $\alpha_{i}$ and $\eta_{i}$ with $\mathrm{Mg}$ doping level is presented as a solid line in Fig. 6. The model, based on drift diffusion theory, predicts a decrease of $j_{t h}$ and an increase of SE for low EBL thicknesses. Surprisingly, the experimentally measured values show an opposite trend to the presented model.
To correctly describe the experiment we propose an additional effect: tunneling of electrons through EBL. If the EBL thickness is insufficient, a part of the electron current might tunnel even though the height of the energetic barrier remains constant (shown by an arrow in Fig. 7). A simple model of reduction of $\eta_{i}$ by tunneling is described by

$$
\eta_{i}^{\prime}=\eta_{i}(1-T),
$$

where $T$ is tunneling probability. In general, it could be described also as an exponential function dependent on the barrier thickness $d$, i.e.,:

$$
T=e^{-k d} \text {. }
$$

The model including the change of $\eta_{i}^{\prime}$ and $\alpha_{i}$ with EBL thickness is presented in Fig. 6, as a dotted line. The fitting parameter is $k=0.16$. This concept qualitatively explains the drop of SE and the increase of $j_{t h}$ observed for low EBL thickness, indicating also that the drift-diffusion model fails at thicknesses below $20 \mathrm{~nm}$. Further work has to be done to verify whether the $20 \mathrm{~nm}$ EBL is sufficient to prevent tunneling.

\section{Summary}

The influence of $\mathrm{Mg}$ doping and thickness of electron blocking layer on threshold current density and slope efficiency of InGaN LDs was studied. It was found that $\mathrm{Mg}$ is responsible for optical losses in LDs grown by PAMBE. Decrease of $\mathrm{Mg}$ doping in EBL improves the performance of studied LDs. However, if the $\mathrm{Mg}$ doping is too low, a decrease of injection efficiency is observed. Additionally, a strong overflow by means of tunneling of electrons through EBL was proposed to describe the experimentally observed change of $j_{t h}$ and SE in samples with thin EBL. Estimating the optimum $\mathrm{Mg}$ doping level and EBL thickness brings us one step further to the development of reliable high power InGaN laser diodes.

\section{Acknowledgments}

This work has been partially supported by TEAMTECH POIR.04.04.00-00-210C/16-00 project of the Foundation for Polish Science co-financed by the European Union under the European Regional Development Fund the National Centre for Research and Development grants LIDER/29/0185/L-7/15/NCBR/2016 and LIDER/35/0127/L-9/17/NCBR/2018.

\section{References}

[1] J.J. Wierer, J.Y. Tsao, D.S. Sizov, Laser Photon Rev. 7, 963 (2013).

[2] U.T. Schwarz, E. Sturm, W. Wegscheider, Appl. Phys. Lett. 83, 4095 (2003).

[3] M. Kuramoto, C. Sasaoka, N. Futagawa, M. Nido, A.A. Yamaguchi, Phys. Status Solidi A 192, 329 (2002). 
[4] M. Kawaguchi, O. Imafuji, S. Nozaki, H. Hagino, S. Takigawa, T. Katayama, T. Tanaka, Proc. SPIE 9748, 974818 (2016).

[5] M. Martens, C. Kuhn, T. Simoneit, S. Hagedorn, A. Knauer, T. Wernicke, M. Weyers, M. Kneissl, Appl. Phys. Lett. 110, 081103 (2017)

[6] Ohad Westreich, Moti Katz, Gil Atar, Yossi Paltiel, Noam Sicron, Appl. Phys. Lett. 111, 022103 (2017).

[7] D. Sizov, R. Bhat, C. Zah, J. Appl. Phys. 113 203108 (2013).

[8] S. Uchida, M. Takeya, S. Ikeda, T. Mizuno, T. Fujimoto, O. Matsumoto, S. Goto, T. Tojyo, M. Ikeda, IEEE J. Sel. Top. Quant. Electron. 9 , 1252 (2003).

[9] E. Kioupakis, P. Rinke, C.G.V. de Walle, Appl. Phys. Expr. 3, 082101 (2010).

[10] E. Kioupakis, P. Rinke, A. Schleife, F. Bechstedt, C.G. Van de Walle, Phys. Rev. B 81, 241201(R) (2010).

[11] G. Muziol, H. Turski, M. Siekacz, P. Wolny, S. Grzanka, E. Grzanka, P. Perlin, C. Skierbiszewski, Appl. Phys. Expr. 8, 032103 (2015).

[12] D.K. Gaskill, L.B. Rowland, K. Doverspike, in: Properties of Group III Nitrides, Ed. J.H. Edgar, EMIS Datareviews Series, INSPEC 1995, p. 101.
[13] W. Götz, N.M. Johnson, C. Chen, H. Liu, C. Kuo, W. Imler, Appl. Phys. Lett. 68, 3144 (1996).

[14] G. Muziol, M. Siekacz, K. Nowakowski-Szkudlarek, M. Hajdel, J. Smalc-Koziorowska, A. FeduniewiczŻmuda and E. Grzanka, P. Wolny, H. Turski, P. Wiśniewski, P. Perlin, C. Skierbiszewski, Mater. Sci. Semicond. Proc. 91, 387 (2019).

[15] G. Muziol, M. Hajdel, M. Siekacz, K. Szkudlarek, S. Stanczyk, H. Turski, C. Skierbiszewski, Appl. Phys. Expr. 12, 072003 (2019).

[16] C. Skierbiszewski, G. Muziol, K. NowakowskiSzkudlarek, H. Turski, M. Siekacz, A. FeduniewiczZmuda, A. Nowakowska-Szkudlarek, M. Sawicka, P. Perlin, Appl. Phys. Expr. 11, 034103 (2018).

[17] C. Skierbiszewski, H. Turski, G. Muziol, M. Siekacz, M. Sawicka, G. Cywiński, Z.R. Wasilewski, S. Porowski, J. Phys. D Appl. Phys. 47, 073001 (2014).

[18] STR, SiLENSe 5.4 package.

[19] G. Muziol, H. Turski, M. Siekacz, S. Grzanka, P. Perlin, C. Skierbiszewski, Appl. Phys. Expr. 9, 092103 (2016).

[20] G. Muziol, H. Turski, M. Siekacz, P. Wolny, J. Borysiuk, S. Grzanka, P. Perlin, C. Skierbiszewski, Opt. Expr. 25, 33113-33121 (2017). 